\title{
Registering Hardness Measurement of Neutron-irradiated Low-activation Steels at High Temperatures
}

\author{
Alexander Valentin Brabänder, Julian Bredl, Hans-Christian Schneider, Marc Kamlah \\ Karlsruhe Institute of Technology, Institute for Applied Materials, Karlsruhe, Germany
}

This work shows an ongoing study of the influence of neutron irradiation on the hardness of first wall structure materials. EUROFER97 and boron-doped steels have been neutron-irradiated in the European High Flux Reactor (HFR) with an average dose of up to $16 \mathrm{dpa}$ at temperatures between 250 and $450{ }^{\circ} \mathrm{C}$. The irradiated samples are investigated by the advanced method of a registering hardness measurement that is used to perform experiments at temperatures of up to $450{ }^{\circ} \mathrm{C}$. The first part of this work examines the hardening of EUROFER97 depending on the irradiation temperature at different test temperatures.

In addition, post-irradiation annealing is applied to EUROFER97 and to the boron-doped steels. The different contents of boron lead to different amounts of helium generated by neutron-irradiation. Since post-irradiation annealing is known to enable the neutron-induced Frenkel pairs and nanoscale defects to recombine, the remaining hardening after the annealing process can be attributed mostly to products of transmutation reactions. Thus, the potential as well as the limits of this promising treatment are explored.

Keywords: EUROFER97, Boron-doped, High-temperature Hardness, Post-irradiation Annealing, Neutron-irradiation

\section{Introduction}

To ensure a safe and long-lasting operation of future fusion reactors, the impact of neutron irradiation and high temperatures on the structural materials needs to be known. For this purpose, different steels have been neutron-irradiated inside the HFR in Petten (NL) with an average damage dose of up to 16 displacements per atom (dpa). The irradiated Charpy impact samples were tested in the Fusion Materials Laboratory (FML) of Karlsruhe Institute of Technology [1]. Due to the low abundance of these irradiated samples, versatile investigations and empirical studies are limited. In this work, a new hightemperature hardness testing facility is used to perform further investigations on halves of already tested Charpy impact samples in order to increase the amount of experimental results per specific sample volume. The aim of this work is to investigate the impact of the temperatures during irradiation and testing on the hardness of EUROFER97. Furthermore, the new method of high-temperature hardness measurement enables versatile investigation of the neutron-induced defects themselves. Post-irradiation annealing (PIA) allows neutron-induced Frenkel pairs and nanoscale defects to recombine, leading to a partial recovery of the material's properties. Thus, it is possible to distinguish between the impact of reversible defects like Frenkel pairs and the non-reversible defects caused during the generation of helium on the hardness.

\section{Method}

Registering hardness measurement requires a small sample volume compared to tensile and Charpy impact tests. This is a high advantage regarding the limited abundance of neutron-irradiated samples. The new experimental setup of the KArlsruhe High Temperature Indenter (KAHTI) allows to perform registering hardness measurement at temperatures of up to $650{ }^{\circ} \mathrm{C}$. The schematic setup of KAHTI is shown in figure 1: KAHTI uses an optical displacement measurement (a) to determine the motion of the indenter (b) and the sample (c) separately via Digital Image Correlation (DIC). This excludes all thermal influences on the load-displacement curve and provides a resolution down to $100 \mathrm{~nm}$. The resolution of the load cell (d) is $50 \mathrm{mN}$. All experiments in KAHTI are performed inside a vacuum chamber (e) at a pressure of $3^{*} 10^{-6} \mathrm{mbar}$ in order to avoid oxidation of the indenter and the sample. By using this new method, it is possible to perform up to 44 additional registering hardness tests on one half of an already tested Charpy impact sample with a dimension of $3 * 4 * 12 \mathrm{~mm}^{3}$. KAHTI is operated by remote control inside a hot cell facility in order to handle radioactive and toxic samples.[2]

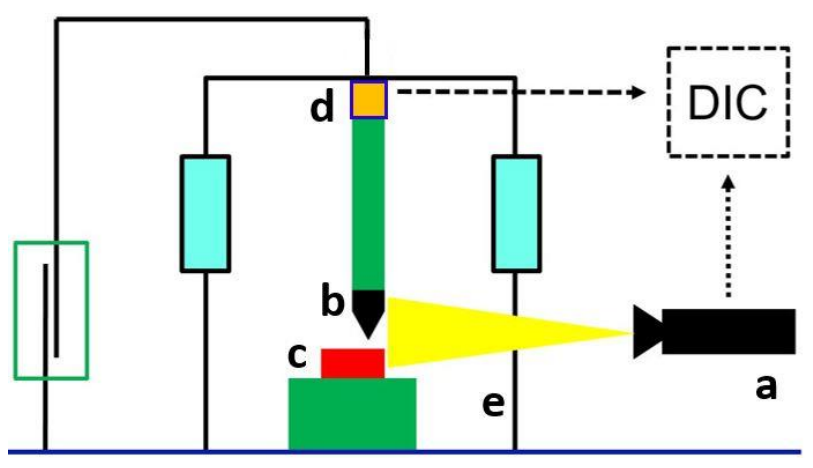

Figure 1: Schematic setup of the KAHTI.

The hardness $H$ is defined as the ratio of the maximum applied force $P_{\max }$ to the area $A$ of the remaining indent. The area function $A(h)$ depends on the shape of the indenter and the indentation depth $h$.

$$
H=\frac{P_{\max }}{A(h)}
$$

In the present work, two different shapes are used: A four-sided pyramid with an opening angle of $136^{\circ}$, the so- 
called Vickers pyramid, and a sphere-shaped Brinell indenter whose sphere has a radius of $200 \mu \mathrm{m}$.

\section{Material and Irradiation Conditions}

Reduced Activation Ferritc/Martensitic (RAFM) steels are the structure materials destined for future fusion reactors. One promising candidate is $9 \% \mathrm{Cr}-\mathrm{WVTa}$ EUROFER97. This study investigates EUROFER97 in the as-received condition of $980{ }^{\circ} \mathrm{C} / 0.5 \mathrm{~h}$ and $760{ }^{\circ} \mathrm{C} / 1.5 \mathrm{~h}$ [1][3]. The primary defects caused by neutron irradiation are, on the one hand, knocked-out lattice atoms and displacement cascades forming Frenkel pairs [4][5]. Depending on the microstructure and temperature during irradiation, these point defects develop into nanoscaled precipitates, dislocation loops as well as vacancy clusters and voids [6]. Those defects hinder the deformation-induced motion of dislocations, causing hardening and embrittlement of the material. On the other hand, a neutron caught in the nucleus of an atom forms an unstable and therefore radioactive isotope, and consequently, transmutation takes place. The decay of unstable isotopes can generate helium, leaving cavities at the lattice site of the parent nuclide. The most prominent reaction forming helium is ${ }^{10} \mathrm{~B}(\mathrm{n}, \alpha)^{7} \mathrm{Li}$ [5][7]. Here, boron transmutes and subsequently decays into lithium and helium. In contrast to defects based on knock-out atoms, defects like helium and cavities are non-reversible.

For representative investigations of the neutroninduced defects during application, EUROFER97 was irradiated with fast and thermal neutrons of the fission source HFR. As the He/dpa ratio is far below the one caused by fusion neutrons, the alloy of EUROFER97 doped with different amounts of boron was included in the irradiation. These Accelerated Degradation Steels (ADS) contain $82 \mathrm{wppm}{ }^{\text {nat }} \mathrm{B}$ (ADS2), $83 \mathrm{wppm}{ }^{10} \mathrm{~B}$ (ADS3) and $1160 \mathrm{wppm}{ }^{10} \mathrm{~B}$ (ADS4) [8]. The irradiation temperatures for particular samples were between $250{ }^{\circ} \mathrm{C}$ and $450{ }^{\circ} \mathrm{C}$. During irradiation on 771 full power days, an average damage dose of $16 \mathrm{dpa}$ was reached [1]. The initial ${ }^{10} \mathrm{~B}$ is assumed to be burned up completely, leading to a helium content of 83 appm in ADS2, 415 appm in ADS3 and 5800 appm in ADS4. The helium content in irradiated EUROFER97 is smaller than 10 appm.[8]

\section{Experimental Procedure}

\subsection{Vickers Hardness}

Registering hardness tests on neutron-irradiated samples above room temperature have not been reported up to date. Thus, in the first part of this work, the hardness of neutron-irradiated EUROFER97 is investigated at high temperatures. The aim is to scan the change of hardness over a temperature range between $250{ }^{\circ} \mathrm{C}$ and $450{ }^{\circ} \mathrm{C}$ for different irradiation temperatures. Halves of tested EUROFER97 Charpy impact samples are prepared by grinding and polishing. Samples with irradiation temperatures of $250{ }^{\circ} \mathrm{C}, 300{ }^{\circ} \mathrm{C}, 350{ }^{\circ} \mathrm{C}, 400{ }^{\circ} \mathrm{C}$ and $450^{\circ} \mathrm{C}$ are available. An additional unirradiated sample of EUROFER97 is used as a reference. All samples are tested at their particular irradiation temperatures. Additional tests at room temperature and at $250{ }^{\circ} \mathrm{C}$ are carried out for all samples to investigate the dependency of hardness on the test temperature. For the aim of a good comparability, all tests are performed with a Vickers indenter tip. For test temperatures $\leq 350{ }^{\circ} \mathrm{C}$, an indenter tip made of diamond is used. For temperatures $>350{ }^{\circ} \mathrm{C}$, the tip is made of sapphire in order to avoid reactions between indenter and steel. The standardized method Vickers Hardness 5 (HV 5) with a maximum load of $49 \mathrm{~N}$ $(=5 \mathrm{kp})$ is applied. This load ensures an indentation volume over several grains and thus representative results. The hardness value is derived from the remaining indentation depth $h$ gained from the recorded loaddisplacement curve.

\subsection{Post-irradiation Annealing}

The second part of this work focuses on the possibility of recovering the material properties by a heat treatment. Therefore, Post Irradiation Annealing (PIA) is applied to neutron-irradiated EUROFER97 and ADS2-4. It is reported in [10] that a heat treatment enables the lattice atoms to move, leading to a recombination of Frenkel pairs and nanoscale defects, whereas defects caused by the generation of helium stay inside the lattice. In this work, all samples are annealed for $3 \mathrm{~h}$ at $550{ }^{\circ} \mathrm{C}$. This temperature is not assumed to influence the grain size, since the tempering temperature is at $760{ }^{\circ} \mathrm{C}$, see also [9]. To differentiate between the impact of the two types of defects on the hardness, samples with the highest density of defects are favorably. The density of defects is prior estimated from the reported strengthening in [5] and [8]. Thus, the most suitable samples available at the FML is EUROFER97 and ADS4 irradiated at $300{ }^{\circ} \mathrm{C}$, and ADS2 and ADS3 irradiated at $250{ }^{\circ} \mathrm{C}$.

For representative results, two halves of the same particular sample are investigated. One half is investigated in the as-irradiated state, the second one is investigated after PIA. Two additional samples of unirradiated EUROFER97, with and without a PIA process, are investigated as references. The heat treatment is carried out inside KAHTI under vacuum of $5 * 10^{-6}$ mbar. To ensure a good comparability, all samples are tested at room temperature. A Brinell shape indenter with a radius of $200 \mu \mathrm{m}$ is used. The maximum applied load is $40 \mathrm{~N}$ in order to get representative results. The Brinell Hardness (HB) is derived from the remaining indentation depth $h$ in the load-displacement curve.

\section{Results}

\subsection{Vickers Hardness}

All Vickers hardness tests on EUROFER97 are performed three or four times. The average values with the associated error bars are shown in figure 2 . Regarding the values measured at room temperature, a hardening from HV 308 of the unirradiated sample to $\mathrm{HV} 413$ of the sample irradiated at $250{ }^{\circ} \mathrm{C}$ is observed. An even higher increase of the hardness with HV 480 is measured at the sample irradiated at $300{ }^{\circ} \mathrm{C}$. This is the maximum measured hardening concerning the irradiation temperature. With a higher irradiation temperature of $350{ }^{\circ} \mathrm{C}$, the hardening decreases again to HV 366. The sample irradiated at $400{ }^{\circ} \mathrm{C}$ shows HV 333. The sample with the highest irradiation temperature of $450{ }^{\circ} \mathrm{C}$ shows HV 321, which 
is almost the original hardness of the unirradiated sample. The hardness of all samples shows a similar dependency to the test temperature. Higher test temperatures clearly soften the material.

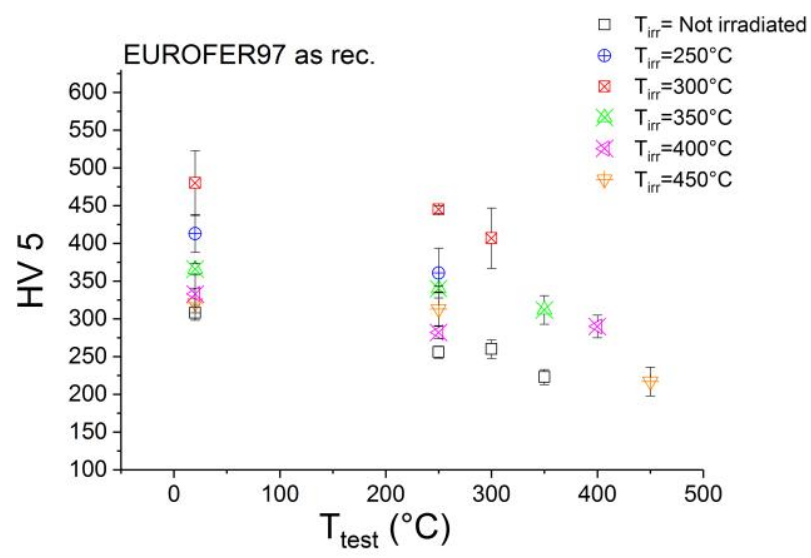

Figure 2: Vickers hardness of EUROFER97 after irradiation with neutrons at $250{ }^{\circ} \mathrm{C}, 300{ }^{\circ} \mathrm{C}, 350{ }^{\circ} \mathrm{C}, 400{ }^{\circ} \mathrm{C}$ and $450{ }^{\circ} \mathrm{C}$ tested at different temperatures. An unirradiated sample is shown as a reference.

The hardness in figure 2, measured at the particular irradiation temperature, represents the hardness under application conditions. For this reason, these values are presented separately in figure 3 . For comparison, the yield stresses $R_{p 0.2}$ from tensile tests given in [11] are shown here as well.

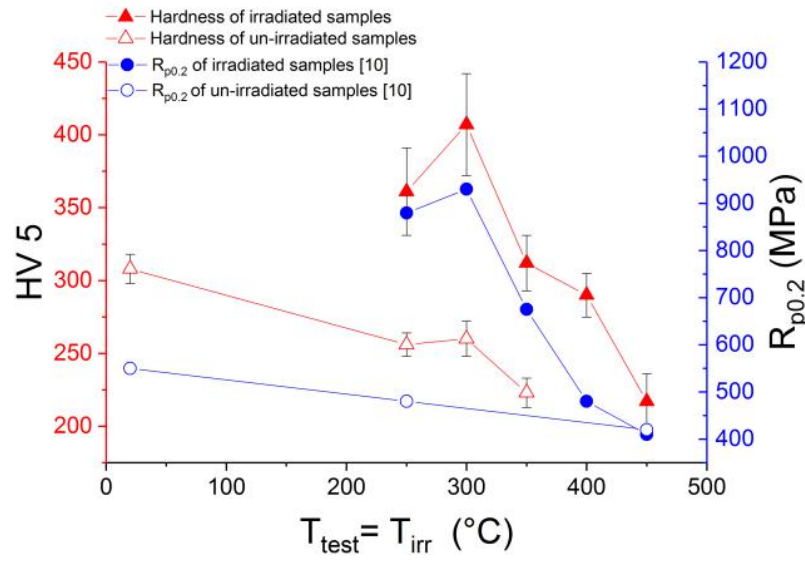

Figure 3: Vickers hardness of EUROFER97 samples tested at the particular irradiation temperature (triangle, solid). Values of yield stresses $R_{p 0.2}$ from [11] are shown as a comparison (circle, solid). As a reference, results of unirradiated samples are shown (open symbols).

\subsection{Post-irradiation Annealing}

Figure 4 displays the results of the post-irradiation annealing experiments. All results rely on five experiments and the error is in the order of $10 \mathrm{HB}$. Compared to the unirradiated EUROFER97, the irradiation-induced defects cause a hardening of $43 \%$ in EUROFER97, $48 \%$ in ADS2, $51 \%$ in ADS3 and $57 \%$ in ADS4. The PIA causes a significant recovery of the materials' hardness. Compared to the unirradiated but annealed EUROFER97, the remaining hardening is $1.5 \%$ for EUROFER97, $9 \%$ for ADS2 and $10 \%$ for ADS3.
Remarkably, ADS4 in the annealed state shows a $14 \%$ lower hardness than the not irradiated and annealed EUROFER97.

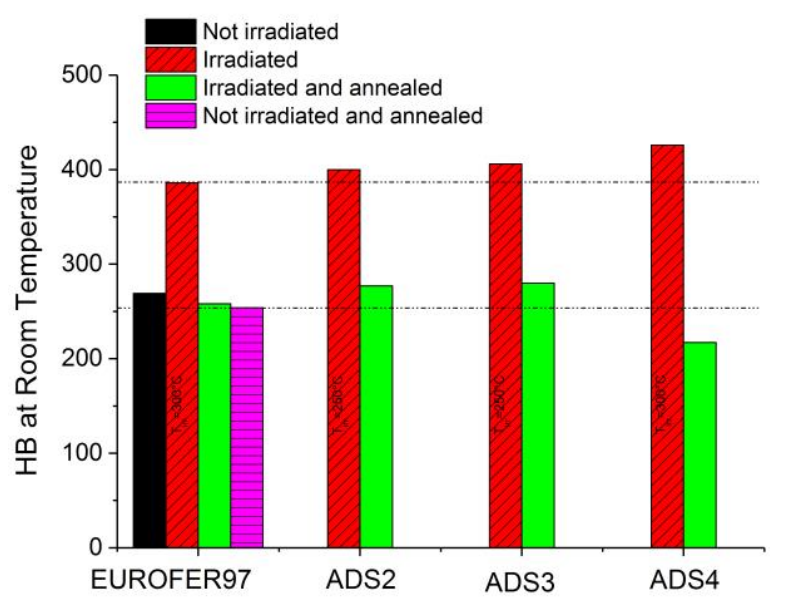

Figure 4: Brinell hardness of irradiated samples, and irradiated samples after PIA. Brinell hardness of unirradiated as well as unirradiated and annealed samples are shown as a reference.

\section{Discussion}

The experimental results of Vickers hardness in figure 2 show a clear dependence on the irradiation temperature. The hardness increases with an increasing irradiation temperature to a maximum at $300{ }^{\circ} \mathrm{C}$. At higher irradiation temperatures, the resulting hardness decreases drastically. As it was shown before in [13], hardness is correlated to the defect density, because defects hinder dislocation motion. Regarding the results in figure 2 it can be assumed that the defect density collapses almost completely above $350{ }^{\circ} \mathrm{C}$. Here, the majority of reversible defects become mobile, dissociate or extinguish by recombination. This leads to a smaller defect density. Therefore, almost original hardness is observed. Compared to that, the test temperature has a minor influence on the hardness. The change in hardness due to the test temperature shows almost the same trend for all samples.

In figure 3 the hardness values, measured at the particular irradiation temperature of the samples, are extracted from figure 2 . Here, the hardness correlates well with the measured yield stresses reported by MaternaMorris et al. in [11]. Since yield stress is known to be related to the hardness of unirradiated materials at room temperature, the presented results verify this relation for irradiated samples at elevated temperatures. Thus, a correlation between yield stress and hardness measurements might be possible for further investigations of irradiated samples with KAHTI.

The measured results in figure 4 show the hardening after irradiation caused by both types of defects, as well as that the hardness after PIA is affected by a reduced ratio of reversible to non-reversible defects. In EUROFER97, the post-irradiation annealing leads to an almost complete recovery of the hardening. The results of ADS2 and ADS3 are more representative in terms of helium generation caused by a fusion source [8]. Here, the samples with 
helium contents of 83 appm and 415 appm cause a higher remaining hardness. Since their remaining hardness does not show a significant difference, it can be assumed that the hardness is not a linear function of the helium generation. However, the impact of the reversible defects is higher compared to that of the non-reversible ones. Nevertheless, non-reversible defects, e.g. in ADS2 and ADS3 after PIA, hinder the motion of dislocations. Further investigations of the microstructure are necessary to check if the annealing process enables agglomeration and diffusion of helium to the grain boundaries, which clearly would enhance the structural damage.

The hardness of ADS4 after PIA is lower compared to that of all other materials investigated. The higher concentrations of non-reversible defects might segregate and sum up leading to a weakening of the microstructure. Additionally, the high content of boron leading to a high amount of precipitations has to be considered. Here, the high presence of ${ }^{10} \mathrm{~B}$ leads to the formation of big cavities [12]. Thus, the results of ADS4 might not be representative of the damage caused by the neutron spectrum of a fusion reactor. Regarding the results found by Schneider et al. [10] the DBTT of neutron-irradiated ADS2-4 samples is around or well above room temperature even after an annealing process. If a PIA is applied to components of future fusion reactors, the nonreversible defects have to be considered.

\section{Conclusion}

The presented results confirm KAHTI as a reliable characterization method for high-temperature application. Its high ratio of experimental results per specific sample volume is a big advantage for the investigation of neutronirradiated samples.

Hardness measurements of neutron-irradiated EUROFER97 causes a maximum hardening at an irradiation temperature of $300{ }^{\circ} \mathrm{C}$. With increasing test temperature, all samples soften. The results of Vickers hardness correlate well with results of tensile tests from [10]. Therefore, a determination of $R_{p 0.2}$ from hardness measurements of neutron-irradiated samples at high temperatures is possible.

Further, the results of post-irradiation annealing show partial recovery of the hardness. Increasing hardness can be correlated to the defect density reported by [12] and [13]. The increasing amount of transmutation reactions products as non-reversible defects, correlate well with increasing hardness, as well as with increasing remaining hardness after PIA. Above a certain amount of generated helium, e.g. in ADS4, the material seems to suffer from general structural damages as it clearly weakens. This is assumed to indicate either segregation of the helium or an increasing quantity of cavities from the parent nuclide. Therefore, transmutation reactions are assumed as the main limiting factor for the effect of PIA. Additional TEM investigations are needed to finally prove the proposed explanations of the different hardening behaviors.

\section{References}

[1] Gaganidze, E., et al. "Irradiation programme HFR phase IIb-SPICE. Impact testing on up to $16.3 \mathrm{dpa}$ irradiated RAFM steels. Final report for task TW2-TTMS 001bD05.". Forschungszentrum Karlsruhe, Wissenschaftliche Berichte No. FZKA-7371 (2008).

[2] Albinski, B., et al. "A new high-temperature indentation device for characterization of materials for fusion applications." Journal of Nuclear Materials 442.1-3 (2013): 865-868.

[3] Petersen, C., et al. "Mechanical properties of reduced activation ferritic/martensitic steels after European reactor irradiations." Proc. of 21st IAEA Fusion Energy Conference, Chengdu, China. (2006).

[4] Kelly, B. T., et al. "Irradiation damage in graphite due to fast neutrons in fission and fusion systems." IAEA Tecdoc 1154 (2000).

[5] Klimenkov, M.,et al. "Characterization of radiation induced defects in EUROFER 97 after neutron irradiation." Journal of Nuclear Materials 417.1-3 (2011): 124-126.

[6] Knaster, J., et al. "Materials research for fusion." Nature Physics 12.5 (2016): 424.

[7] Gaganidze, E., et al. "Mechanical properties and TEM examination of RAFM steels irradiated up to $70 \mathrm{dpa}$ in BOR-60." Journal of Nuclear Materials 417.1-3 (2011): 93-98

[8] Materna-Morris, E., et al. "Effect of helium on tensile properties and microstructure in 9\% Cr-WVTa-steel after neutron irradiation up to 15 dpa between 250 and $450{ }^{\circ} \mathrm{C} . "$ Journal of Nuclear Materials 386 (2009): 422 425.

[9] Graf, P., et al. "Der Einfluss von Bor auf die Gefugeeigenschaften von martensitischen 9\%Chromstahlen." SONDERBAENDE DER PRAKTISCHEN METALLOGRAPHIE (2004): 71-76.

[10] Schneider, H-C., et al. "Repeatability of irradiation damage and of recovery by post-irradiation annealing of EUROFER base steels." Fusion Engineering and Design 124 (2017): 1019-1023.

[11] Materna-Morris, E., et al. "Tensile and low cycle fatigue properties of EUROFER97-steel after 16.3 dpa neutron irradiation at 523, 623 and 723 K." Journal of Nuclear Materials 442.1-3 (2013): 62-66.

[12] Klimenkov, M., et al. "Helium bubble morphology of boron alloyed EUROFER97 after neutron irradiation." Journal of Nuclear Materials 442.1-3 (2013): S52-S57.

[13] Klimenkov, M., et al. "Helium influence on the microstructure and swelling of $9 \% \mathrm{Cr}$ ferritic steel after neutron irradiation to 16.3 dpa." Journal of Nuclear Materials 453.1-3 (2014): 54-59. 